\title{
Preheating, Thermalization and Supergravity
}

\author{
Lev Kofman \\ CITA, University of Toronto, M5S 3H8, Canada
}

\begin{abstract}
This constribution collects new recent results on preheating after inflation. We discuss tachyonic preheating in the SUSY motivated hybrid inflation; development of equilibrium after preheating; theory of fermionic preheating and the problem of gravitino overproduction from preheating.
\end{abstract}

\section{Introduction}

The best-fit universe is the flat FRW universe with scale-free scalar metric fluctuations. The model is in agreement with the observations of the cosmic microwave background radiation (CMB) anisotropy, the large scale structure (LSS) of the universe and the tests of the global geometry such as the high redshift supernovae (SNe). The theoretical scenario of the expanding universe includes a very early stage of inflation, subsequent stage of particle creation during preheating and the setting of the thermal equilibrium. The resulting hot expanding universe passes through a sequence of crucial stages. As the universe expands, it opens up for the observational tests, beginning "upward" from the big bang nucleosynthesis $(\mathrm{BBN})$, see the left panel of Figure 1.

Although inflation is an essential part of the cosmological paradigm, from the particle physics perspective it is not easy to construct a satisfactory model

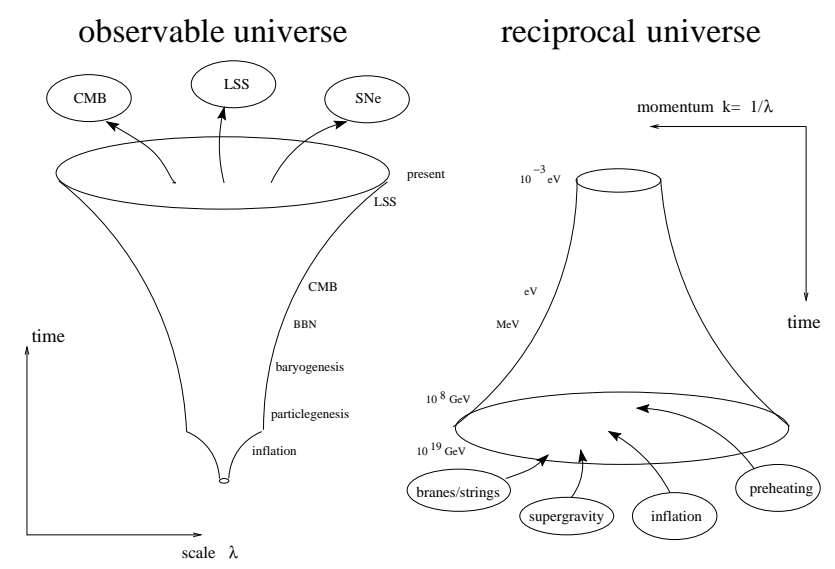

Fig. 1. Sketch of an expanding universe where the wavelengths $\lambda(t)$ are proportional to a scale factor $a(t)$ and "reciprocal" universe where the momenta $k(t) \sim 1 / \lambda(t)$ are inversly proportional to $a(t)$. 
of the very early universe including inflation. The right panel of Figure 1 shows a theoretical "reciprocal" universe where the momenta $k \sim 1 / \lambda$ are increasing backwards in time. The reciprocal universe at the earliest times at high momenta opens up for theoretical possibilities of the early universe physics, including recent developments in brane cosmology, string motivated cosmology, supergravity in cosmology etc.

Fundamental M-theory should encompass both supergravity and string theory. At present the low-energy phenomenology is described by the $N=1 d=4$ supergravity. Some preferable choices of the Kähler potentials, superpotentials and Yang-Mills couplings hopefully will be selected at the level of the fundamental theory. Until the fundamental theory of all interactions is well understood, one may try to address the issues of the eary universe cosmology in the context of the most general phenomenological $N=1$ supergravity-Yang-Mills-matter theory. This, in fact, was the case during the last almost 20 years. A rather lengthy $N=1$ phenomenological supergravity Lagrangian begins with the terms

$$
\begin{aligned}
e^{-1} \mathcal{L} & =-\frac{1}{2} M_{P}^{2} R-\hat{\partial}_{\mu} \Phi^{i} \hat{\partial}^{\mu} \Phi_{i}+e^{K}\left(\mathcal{D}^{i} W \mathcal{D}_{i} W-3 \frac{W W^{*}}{M_{P}^{2}}\right) \\
& -\bar{\chi}_{j} \not \supset \chi^{i}-\bar{\chi}^{i} \not \supset \chi_{j}-\left(e^{K / 2} \mathcal{D}^{i} \mathcal{D}^{j} W \bar{\chi}_{i} \chi_{j}+\text { h.c. }\right) \\
& -\frac{1}{2} \bar{\psi}_{\mu} R^{\mu}+\left(\frac{1}{2} e^{K / 2} W \bar{\psi}_{\mu R} \gamma^{\mu \nu} \psi_{\nu R}+\bar{\psi}_{\mu L} \hat{\partial} \Phi^{i} \gamma^{\mu} \chi_{i}+\bar{\psi}_{R} \chi_{i} e^{K / 2} \mathcal{D}^{i} W+\text { h.c. }\right) \\
& +\ldots
\end{aligned}
$$

A particular choice of the form of the Lagrangian is motivated and notations are given in [5. In Eq. (11) $K$ is the Kähler potential, $\Phi^{i}$ is the complex conjugate of $\Phi_{i}$.

According to the inflationary scenario, the Universe initially expands quasiexponentially in a vacuum-like state without entropy or particles. At the stage of inflation, all energy is contained in a classical slowly moving fields $\Phi$ in the inflaton sector. The last term of the 1st line of (1) is the scalar potential $V\left(\Phi_{i}\right)$. The equations of motion based on the first line should describe inflation, which is a challenging problem by itself. The Lagrangian (11) contains also other fields which give subdominant contributions to gravity. The Friedmann equation for the scale factor $a(t)$ and the Klein-Gordon equation for $\Phi(t)$ determine the evolution of the background fields. In the chaotic inflation models, soon after the end of inflation, an almost homogeneous inflaton field $\Phi(t)$ coherently oscillates with a very large amplitude of the order of the Planck mass around the minimum of its potential. This scalar field can be considered as a coherent superposition of inflatons with zero momenta. The amplitude of oscillations gradually decreases not only because of the expansion of the universe, but also because energy is transferred to particles created by the oscillating field. At this stage we shall recall the rest of the fundamental Lagrangian which includes all the fields interacting with inflaton. These interactions lead to the creation of many ultra-relativistic particles from the inflaton. Gradually, the inflaton field decays and transfers all of its energy to the created particles. In this scenario all the matter constituting 
the universe is created from this process of reheating. If the creation of particles is sufficiently slow, the particles would simultaneously interact with each other and come to a state of thermal equilibrium at the reheating temperature $T_{R}$. This gradual reheating can be treated with the perturbative theory of particle creation and thermalization. However, typically particle production from coherently oscillating inflatons occurs not in the perturbative regime but in the non-perturbative regime of preheating [1].

In this contribution I will discuss several problems of preheating after inflation, some of them related to the supergravity. This discussion is based on new results derived in recent papers [3, 5, 8, 6, 6, 2].

\section{Tachyonic Preheating}

Another popular class of inflationary models - hybrid inflation - involve multiple scalar fields $\Phi_{i}$ in the inflaton sector. Previous studies of preheating in hybrid models were concentrated on particle creation by parametric resonance that may occur when homogeneous background fields oscillate around the minimum of the potential. Such parametric resonance may or may not be strong depending on the coupling parameters. However, we recently found [7] that there is strong preheating in hybrid inflation, but its character is quite different from preheating based on parametric resonance. It turns out that typically there is tachyonic instability that appears in a broad class of hybrid inflation models. The backreaction of rapidly generated fluctuations does not allow homogeneous background oscillations to occur because all energy of the oscillating field is transferred to the energy of long-wavelength scalar field fluctuations within a single oscillation. However, this does not preclude the subsequent decay of the Higgs and inflaton inhomogeneities into other particles, and thus reheating without parametric resonance.

Consider the simple potential for the two-field hybrid inflation is

$$
V(\phi, \sigma)=\frac{\lambda}{4}\left(\sigma^{2}-v^{2}\right)^{2}+\frac{g^{2}}{2} \phi^{2} \sigma^{2},
$$

where we used notations $\Phi_{1}=\phi, \Phi_{2}=\sigma$. Inflation in this model occurs while the homogeneous $\Phi_{1}$ field slow rolls from large $\phi$ towards the bifurcation point at $\phi=\frac{\sqrt{\lambda}}{g} v$ (due to the slight lift of the potential in $\phi$ direction). Once $\phi(t)$ crosses the bifurcation point, the curvature of the $\sigma$ field, $m_{\sigma}^{2} \equiv \partial^{2} V / \partial \sigma^{2}$, becomes negative. This negative curvature results in exponential growth of $\sigma$ fluctuations. Inflation then ends abruptly in a "waterfall" manner.

One reason to be interested in hybrid inflation is that it can be implemented in supersymmetric theories. In particular, for illustration we will consider preheating in the supersymmetric F-term inflation as an example of a hybrid model.

The simplest F-term hybrid inflation model (without undesirable domaine walls) is based on a superpotential with three left-chiral superfields $\Phi_{i}=\left(\Phi_{1}, \Phi_{2}, \Phi_{3}\right)$ in the Lagrangian (11)

$$
W=\frac{\sqrt{\lambda}}{2} \Phi_{1}\left(4 \Phi_{2} \Phi_{3}-v^{2}\right)
$$


In this case, the spontaneous breaking of the local (global) $U(1)$ symmetry between the $\Phi_{2}$ and $\Phi_{3}$ fields will lead to gauge (global) string formation.

In global SUSY, using the same notation for superfields and their complex scalar components, this superpotential contributes

$$
V_{\mathrm{F}}=\frac{\lambda}{4}\left|4 \Phi_{2} \Phi_{3}-v^{2}\right|^{2}+4 \lambda\left|\Phi_{1}\right|^{2}\left(\left|\Phi_{3}\right|^{2}+\left|\Phi_{2}\right|^{2}\right) .
$$

to the scalar potential. In general, $\Phi_{3}$ and $\Phi_{3}$ could be (oppositely) charged under a local $U(1)$ symmetry, in which case we should include a D-term, $V_{\mathrm{D}}$, which we negelect here.

In this model, inflation occurs when chaotic initial conditions lead to $\left\langle\left|\Phi_{1}\right|\right\rangle \gg$ $v$. When this happens, the fields $\Phi_{2}$ and $\Phi_{3}$ acquire large effective masses and roll to their local minimum at $\left\langle\Phi_{2}\right\rangle=\left\langle\Phi_{3}\right\rangle=0$. In this limit, the potential (14) becomes $V \approx \frac{\lambda v^{4}}{4}$, which gives rise to a non-vanishing effective cosmological constant. However, this is a false vacuum state; the true vacuum corresponds to $\left\langle\Phi_{2} \Phi_{3}\right\rangle=\frac{v^{2}}{4}$ and $\left\langle\Phi_{1}\right\rangle=0$. The slow-roll potential drives the evolution of the inflaton towards its true VEV. When its magnitude reaches the value $\left\langle\left|\Phi_{\mathrm{c}}\right|\right\rangle=\frac{v}{2}$ spontaneous symmetry breaking occurs.

For further discussion of symmetry breaking in this model, let us rewrite (仼) in terms of polar fields: $\Phi_{3}=\left|\Phi_{3}\right| e^{i \theta}, \Phi_{2}=\left|\Phi_{2}\right| e^{i \bar{\theta}}$. The potential becomes

$$
V_{\mathrm{F}}=\frac{\lambda}{4}\left(16\left|\Phi_{2}\right|^{2}\left|\Phi_{3}\right|^{2}-8 v^{2}\left|\Phi_{2}\right|\left|\Phi_{3}\right| \cos (\theta+\bar{\theta})+v^{4}\right)+4 \lambda\left|\Phi_{2}\right|^{2}\left(\left|\Phi_{2}\right|^{2}+\left|\Phi_{3}\right|^{2}\right) .
$$

At the stage of symmetry breaking, when $\left\langle\left|\Phi_{2} \Phi_{3}\right|\right\rangle$ begins to move away from zero, the absolute phase $\operatorname{Arg}\left(\Phi_{2} \Phi_{3}\right)=\theta+\bar{\theta}$ acquires a mass and is forced to zero. Note, however, that the potential is independent of the relative phase, $\theta-\bar{\theta}$, reflecting the $U(1)$ symmetry. Thus, in a quasi-homogeneous patch, the $U(1)$ symmetry allows us to choose the relative phase of the $\Phi_{2}, \Phi_{3}$ fields to be zero without any loss of generality. This choice, combined with the vanishing of the absolute phase, is equivalent to choosing the two complex $\Phi_{2}, \Phi_{3}$ fields to be real. In order to leave canonical kinetic terms, we define $\sigma_{ \pm} \equiv\left|\Phi_{3}\right| \pm\left|\Phi_{2}\right|$. Furthermore, as inflation has left the inflaton homogeneous across all the patches, we may choose it to be real: $\phi \equiv \sqrt{2}\left|\Phi_{1}\right|$.

In terms of these three real fields, the potential now becomes

$$
V_{\mathrm{F}}=\frac{\lambda}{4}\left(\sigma_{+}^{2}-\sigma_{-}^{2}-v^{2}\right)^{2}+\lambda \phi^{2}\left(\sigma_{+}^{2}+\sigma_{-}^{2}\right) .
$$

In the symmetric phase, when $\sigma_{ \pm}=0$, the $\sigma$ fields have an effective masssquared: $m_{ \pm}^{2}(\phi)=\lambda\left(2 \phi^{2} \mp v^{2}\right)$. We can now see that spontaneous symmetry breaking occurs in this model exactly as in the two field model (2). For $\phi<$ $\phi_{c}=\frac{v}{\sqrt{2}}$, the $\sigma_{+}$field has a tachyonic mass that triggers symmetry breaking and the end of inflation. On the other hand, the $\sigma_{-}$field has always a large and positive effective mass-squared, pinning it to zero. Thus, during inflation and at the initial stages of symmetry breaking, this model behaves just like the standard two field hybrid model discussed in the last section. We have only to apply the 


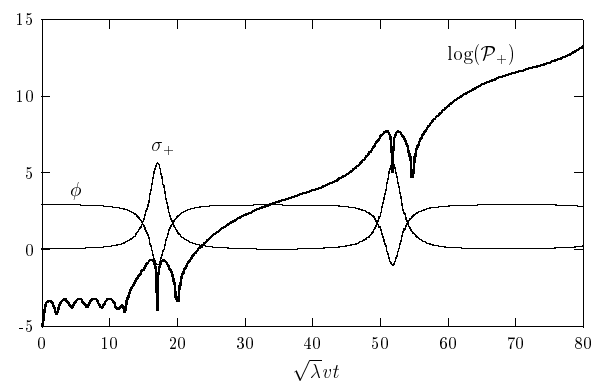

Fig. 2. Evolution of the background fields $\sigma_{+}(t)$ and $\phi(t)$ after symmetry breaking and the $\log$ of $\mathcal{P}_{+}(k)$ for the mode with the momentum $k=0.2 \sqrt{\lambda} v$.

constraint $g^{2}=2 \lambda$ and identify the Higgs field with $\sigma_{+}$. The equations for the homogeneous background components $\phi(t)$ and $\sigma_{ \pm}(t)$ admits simple solution

$$
\phi(t)+\frac{1}{\sqrt{2}} \sigma_{+}(t)=\phi_{c}, \quad \sigma_{-}(t)=0 .
$$

To study preheating in the F-term inflation, we have to analyse evolution of the vacuum fluctuations. Consider vacuum fluctuations in the inflaton sector $\Phi_{i}$ of the theory (5). Usual description in terms of a homogeneous background plus small fluctuations gives us equations for fluctuations around the background solution (7). We define the variances of fields:

$$
\left\langle\left|\sigma_{ \pm}-\left\langle\sigma_{ \pm}\right\rangle\right|^{2}\right\rangle_{\text {ren }}=\int \frac{d^{3} k}{(2 \pi)^{3}}\left[\left|\delta \sigma_{k \pm}(t)\right|^{2}-\left|\delta \sigma_{k \pm}(0)\right|^{2}\right] \equiv \int \frac{d k}{k} \mathcal{P}_{ \pm}(k, t)
$$

and similar for $\phi$ field. Here $\mathcal{P}_{ \pm, \phi}(k, t)$ are the spectra of the fluctuations.

Numerical solutions of the equations for the background fields and for the time evolution of the fluctuations of $\sigma_{+}$for the mode $k$ where their spectrum is maximum are plotted as the bold line at the Figure 2. Notice an enormous exponential growth of the fluctuations within a single background oscillation. Indeed, the amplitude $\mathcal{P}_{+}(k)$ increases by factor $10^{10}$ ! There are two factors which contributes to such a strong instability of fluctuations in the model. First, oscillating background fields are crossing the region with significant negative curvature of the effective potential, which results in tachyonic instability. Second, this region turn out to be a turning point for the background oscillations, where the fields spend significant portion of the oscillation. As a result, tachyonic instability is lasting long enough to make the backreaction of the fluctuations to be significant already within single background oscillation. The regime of background oscillations will even not be settled. Therefore practically from the beginning we have to use the lattice simulations to study nonlinear dynamics of the fields. The results of full lattice simulations in the model are plotted in Figure 3. The simulations showed that the homogeneous fields $\phi$ and $\sigma_{+}$initially followed the classical 
trajectory (7) but, within one oscillation of the inflaton field, fluctuations grew too large to speak meaningfully of the fields as homogeneous oscillators. These fluctuations grew in such a way that $\sigma_{+}=\sigma_{-}{ }^{*}$ almost exactly throughout the simulation. In other words $\operatorname{Re} \delta \sigma_{+}$and $\operatorname{Im} \delta \sigma_{-}$were excited while $\operatorname{Im} \delta \sigma_{+}$and $\operatorname{Re} \delta \sigma_{-}$were not. Because of this we only plot the fields $\phi$ and $\sigma_{+}$.

In [7] we develop a general theory of tachyonic preheating, which occurs due to tachyonic instability in the theories with spontaneous symmetry breaking. Our approach combines analytical estimates with lattice simulations taking into account all backreaction effects. The process of spontaneous symmetry breaking involves transfer of the potential energy into the energy of fluctuations produced due to the tachyonic instability. We show that this process is extremely efficient and requires just a single oscillation of the scalar field falling from the top of the effective potential. We considered preheating in the hybrid inflation scenario, including SUSY motivated F-term and D-term inflationary models.

\section{Development of Equilibrium}

The character of preheating may vary from model to model, e.g. parametric excitation in chaotic inflation [1] and tachyonic preheating in hybrid inflation [7], but its distinct feature remains the same: rapid amplification of one or more bosonic fields to exponentially large occupation numbers. This amplification is eventually shut down by backreaction of the produced fluctuations. The end result of the process is a turbulent medium of coupled, inhomogeneous, classical waves far from equilibrium. Despite the development of our understanding of preheating after inflation, the transition from this stage to a hot Friedmann universe in thermal equilibrium has remained relatively poorly understood. The details of this thermalization stage depend on the constituents of the fundamental Lagrangian (11) and their couplings, so at first glance it would seem that a

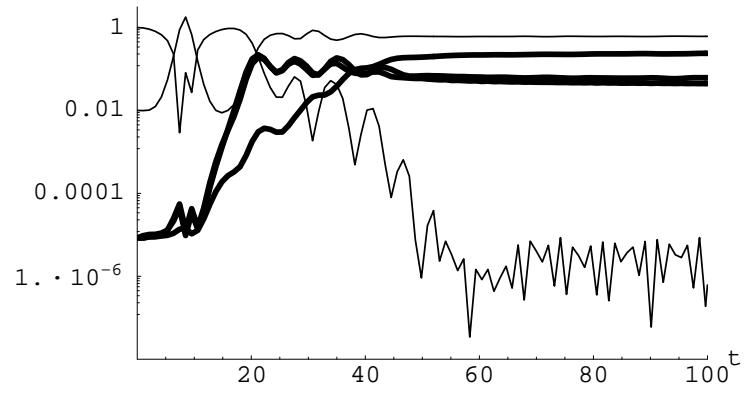

Fig. 3. Means and variances in units of $\phi_{c}$. The squared means $\langle\phi\rangle^{2}$ and $\left\langle\sigma_{+}\right\rangle^{2}$ are ordinary solid lines while the field variances are thick lines. The mean of $\phi$ starts at $\phi_{c}$, oscillates once, and then decays. The mean of $\sigma_{+}$grows in antiphase to $\phi$ and freeses at $\phi_{c}$. 
description of this process would have to be strongly model-dependent. Recently we performed a fully nonlinear study of the development of equilibrium after preheating [6]. We have performed lattice simulations of the evolution of interacting scalar fields during and after preheating for a variety of inflationary models. We have found, however, that many features of this stage seem to hold generically across a wide spectrum of models. Indeed, at the end of preheating and beginning of the turbulent stage $t_{*}$, the fields are out of equilibrium. We have examined many models and found that at $t_{*}$ there is not much trace of the linear stage of preheating and conditions at $t_{*}$ are not qualitatively sensitive to the details of inflation. We therefore expect that this second, highly nonlinear, turbulent stage of preheating may exhibit some universal, model-independent features. Although a realistic model would include one or more Higgs-Yang-Mills sectors, we treat the simpler case of interacting scalars.

We have numerically investigated the processes of preheating and thermalization in a variety of models and determined a set of rules that seem to hold generically. These rules can be formulated as follows (in this section we use notations $\phi=\Phi_{1}$ for the inflaton field and $\chi, \sigma$ for other scalars $\Phi_{i}$ )

1. In many, if not all viable models of inflation there exists a mechanism for exponentially amplifying fluctuations of at least one field $\chi$. These mechanisms tend to excite long-wavelength excitations, giving rise to a highly infrared spectrum.

The mechanism of parametric resonance in single-field models of inflation has been studied for a number of years. This effect is quite robust. Adding additional fields (e.g. $\sigma$ fields) or self-couplings (e.g. $\chi^{4}$ ) has little or no effect on the resonant period. Moreover, in many hybrid models a similar effect occurs due to tachyonic instability. The qualitative features of the fields arising from these processes seem to be largely independent of the details of inflation or the mechanisms used to produce the fields.

2. Exciting one field $\chi$ is sufficient to rapidly drag all other light fields with which $\chi$ interacts into a similarly excited state.

We have seen this effect when multiple fields are coupled directly to $\chi$ and when chains of fields are coupled indirectly to $\chi$. All it takes is one field being excited to rapidly amplify an entire sector of interacting fields. These second generation amplified fields will inherit the basic features of the $\chi$ field, i.e. they will have spectra with more energy in the infrared than would be expected for a thermal distribution.

3. The excited fields will be grouped into subsets with identical characteristics (spectra, occupation numbers, effective temperatures) depending on the coupling strengths.

We have seen this effect in a variety of models. For example in the models (9) which we are going to consider the $\chi$ and $\sigma$ fields formed such a group. In general, fields that are interacting in a group such as this will thermalize much 
more quickly than other fields, presumably because they have more potential to interact and scatter particles into high momentum states.

4. Once the fields are amplified, they will approach thermal equilibrium by scattering energy into higher momentum modes.

This process of thermalization involves a slow redistribution of the particle occupation number as low momentum particles are scattered and combined into higher momentum modes. The result of this scattering is to decrease the tilt of the infrared portion of the spectrum and increase the ultraviolet cutoff of the spectrum. Within each field group the evolution proceeds identically for all fields, but different groups can thermalize at very different rates.

Here we will illustrate these results with a simple chaotic inflation model with a quartic inflaton potential. The inflaton $\phi$ has a four-legs coupling to another scalar field $\chi$, which in turn can couple to two other scalars $\sigma_{1}$ and $\sigma_{2}$. The potential for this model is

$$
V=\frac{1}{4} \lambda \phi^{4}+\frac{1}{2} g^{2} \phi^{2} \chi^{2}+\frac{1}{2} h_{1}^{2} \chi^{2} \sigma_{1}^{2}+\frac{1}{2} h_{2}^{2} \chi^{2} \sigma_{2}^{2}
$$

Preheating in this theory in the absence of the $\sigma_{i}$ fields is well studied. For nonsmall $\frac{g^{2}}{\lambda}$ the field $\chi$ will experience parametric amplification, rapidly rising to exponentially large occupation numbers. In the absence of the $\chi$ field (or for sufficiently small $g$ ) $\phi$ will be resonantly amplified through its own selfinteraction, but this self-amplification is much less efficient than the two-field interaction. The results shown here are for $\lambda=9 \times 10^{-14}$ (for CMB anisotropy normalization) and $g^{2}=200 \lambda$. When we add a third field we use $h_{1}^{2}=100 g^{2}$ and when we add a fourth field we use $h_{2}^{2}=200 g^{2}$.

One of the most interesting variable to calculate is the (comoving) number density of particles of the fields $n(t)$ and their occupation number $n_{k}$. The evolution of the total number density of all particles $n_{t o t}$ is an indication of the physical processes taking place. In the weak interaction limit the scattering of classical waves via the interaction $\frac{1}{2} g^{2} \phi^{2} \chi^{2}$ can be treated using a perturbation expansion with respect to $g^{2}$. The leading four-legs diagrams for this interaction corresponds to a two-particle collision $(\phi \chi \rightarrow \phi \chi)$, which conserves $n_{t o t}$. The regime where such interactions dominate corresponds to "weak turbulence" in the terminology of the theory of wave turbulence. If we see $n_{t o t}$ conserved it will be an indication that these two-particle collisions constitute the dominant interaction. Conversely, violation of $n_{t o t}(t)=$ const will indicate the presence of strong turbulence, i.e. the importance of many-particle collisions. Such higher order interactions may be significant despite the smallness of the coupling parameter $g^{2}$ (and others) because of the large occupation numbers $n_{k}$. Later, when these occupation numbers are reduced by rescattering, the two-particle collision should become dominant and $n_{\text {tot }}$ should be conserved. For a bosonic field in thermal equilibrium with a temperature $T$ and a chemical potential $\mu$ the spectrum of occupation numbers in the limit of classical waves is given by

$$
n_{k} \approx \frac{T}{\omega_{k}-\mu} .
$$




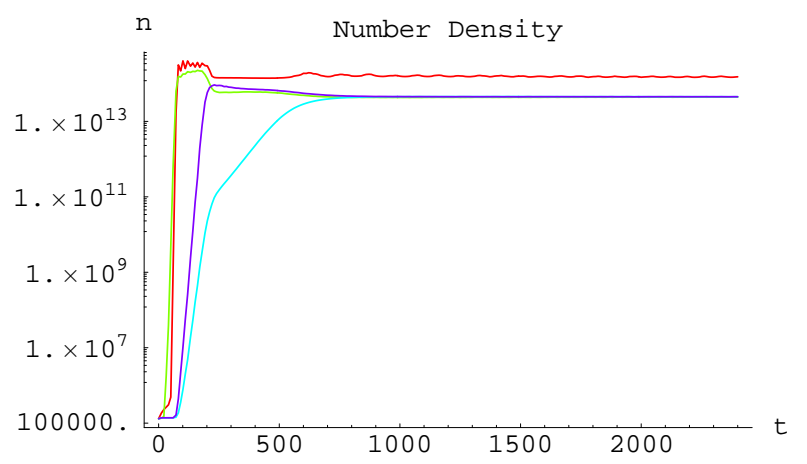

Fig. 4. Time evolution of number density of particles in the model (9). The curves represent $n_{\phi}, n_{\chi}, n_{\sigma_{1}}, n_{\sigma_{2}}$ from top to bottom. Unit of (conformal) time is $a \cdot 10^{-36}$ sec.

Figure 14 shows an exponential increase of $n(t)$ during preheating, followed by a gradual decrease that asymptotically slows down. This exponential increase is a consequence of explosive particle production due to parametric resonance. After preheating the fields enter a turbulent regime. In our simulations we see $n(t)$ decreasing during this stage. This decrease is a consequence of the manyparticle interactions beyond the four legs rescattering.

Figure 5 illustrates the late-time spectrum of particles. Initially spectra are concentrated at the infrared section, which is gradually flattening as it approaches a thermal distribution (10) (i.e. a slope of -1 to 0 depending on the chemical potential and the mass). The spectra of three fields are essentially identical, which leads to

$$
n_{\chi} \approx n_{\sigma 1} \approx n_{\sigma 2}<n_{\phi}
$$

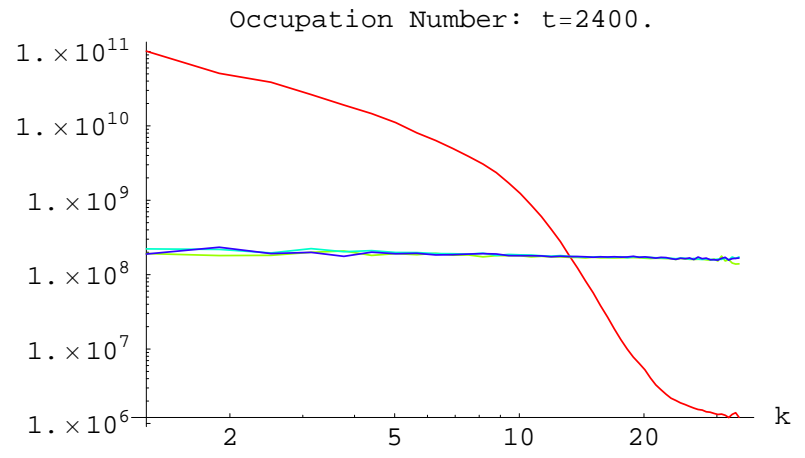

Fig. 5. Occupation number versus momentum for the model (9). The curves represent spectra of particles for the fields $\phi, \chi, \sigma_{1}$ and $\sigma_{2}$ from top to bottom All the fields other than the inflaton have nearly identical spectra 
Another important point is that the interaction of $\chi$ and $\sigma_{i}$ does not affect the preheating of $\chi_{i}$, but does drag $\sigma_{i}$ exponentially quickly into an excited state. The fields $\sigma_{i}$ are exponentially amplified not by parametric resonance, but by their stimulated interactions with the amplified $\chi$ field. Unlike amplification by preheating, this direct decay nearly conserves particle number, with the result that $n_{\chi}$ decreases as $\sigma_{i}$ grow.

Interacting waves of scalar fields constitute a dynamical system. Dynamical chaos is one of the features of wave turbulence. In [6] we address the question how and when the onset of chaos takes place after preheating. To investigate the onset of chaos we have to follow the time evolution of two initially nearby points in the phase space. Consider the theory with the potential (9) with two fields $\phi$ and $\chi$ only (which we collectively denote as $f$ ). Consider two configurations of a scalar field $f$ and $f^{\prime}$ that are identical except for a small difference of the fields at a set of points $x_{A}$. Chaos can be defined as the tendency of such nearby configurations in phase space to diverge exponentially over time. This divergence is parametrized by the Lyapunov exponent for the system, defined as

$$
\lambda \equiv \frac{1}{t} \log \frac{\Delta(t)}{\Delta_{0}}
$$

where $\Delta$ is a distance between two configurations and $\Delta_{0}$ is the initial distance at time 0 . In the context of preheating is is convenient to define $\Delta$ as

$$
\Delta(t) \equiv \sum_{A}\left(\frac{f_{A}^{\prime}-f_{A}}{f_{A}^{\prime}+f_{A}}\right)^{2}+\left(\frac{\dot{f}_{A}^{\prime}-\dot{f}_{A}}{\dot{f}_{A}^{\prime}+\dot{f}_{A}}\right)^{2}
$$

that is well regularized even while the field $\chi$ is being amplified exponentially during preheating. Figure 6 shows the Lyapunov exponent $\lambda$. We see the onset of chaos only at the end of preheating. The plot for the $\phi$ field is nearly identical. The Lyapunov exponents for the fields were $\lambda_{\phi} \approx \lambda_{\chi} \approx 0.2$ (in the units of time

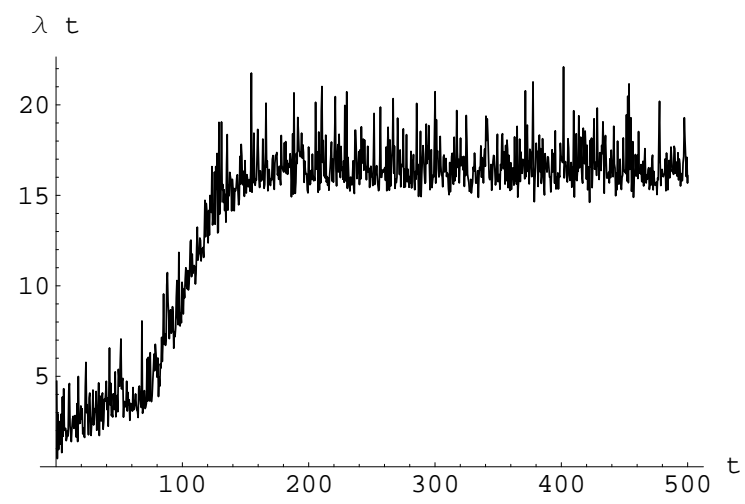

Fig. 6. The Lyapunov exponent $\lambda$ for the fields $\phi$ and $\chi$ using the normalized distance function $\Delta$. 
adopted in the simulation). This corresponds to a very fast onset of chaos around the moment $t_{*}$ where the strong tubulence begins.

The highlights of our study for early universe phenomenology are the following. The mechanism of preheating after inflation is rather robust and works for many different systems of interacting scalars. There is a stage of turbulent classical waves where the initial conditions for preheating are erased. Initially, before all the fields have settled into equilibrium with a uniform temperature, the reheating temperature may be different in different subgroups of fields. The nature of these groupings is determined by the coupling strengths.

\section{Parametric Excitations of Fermions}

The interaction between bosons and inflaton(s) may results in the copious production - preheating - of the bose particles, either due to the parametric resonance as in the chaotic inflation or due to the tachyonic instability as in the hybrid inflation. Preheating of bosons has been studied in details with analytic approximations as well as with the lattice simulations. Consider second line of Eq. (11) and ignore mixing between fermions $\chi$ and gravitino $\psi_{\mu}$ (which corresponds to the rigid SUSY limit). There is interaction between inflaton and fermions. Let us consider a simple Yukawa interaction $h \bar{\psi} \phi \psi$, which should lead to the production of fermions from inflaton oscillations (in this section we use notation $\phi$ for the inflaton field). For fermions, the Pauli exclusion principle prohibits the occupation number from exceeding 1. For this reason, it has been silently assumed that fermions are created in the three-legs perturbative process $\phi \rightarrow \bar{\chi} \chi$ where individual inflatons decay independently into pairs of $\chi$-particles. Following [2] let us, however, consider the Dirac equation for a massless quantum Fermi field $\chi(t, \boldsymbol{x})$ intracting with the background inflaton

$$
\left[\gamma^{\mu} \nabla_{\mu}+h \phi(t)\right] \chi=0
$$

where $\nabla_{\mu}$ is the derivative with the spin connection. Here, similar to the bosonic case, the inflatons producing fermions also act not as individual particles but as a coherently oscillating field $\phi(t)$. Indeed, the equation for the eigenfunctions of the quantum fluctuations in this theory can be reduced to a second-order equation for an auxillary field $X(t, \boldsymbol{x})$, so that $\Psi=\left[\gamma^{\mu} \nabla_{\mu}+h \varphi\right] X$. The eigenmodes of the auxillary field have the form $X_{k}(t) e^{+i \mathbf{k} \cdot \mathbf{x}} R_{r}$, where the $R_{r}$ are eigenvectors of the Dirac matrix $\gamma^{0}$ with eigenvalue +1 (we are using the representation of gamma matrices where $\left.\gamma_{0}=\operatorname{diag}(i, i,-i,-i)\right)$. The temporal part of the eigenmode obeys an oscillator-like equation with a complex frequency which depends periodically on time

$$
\ddot{X}_{k}+\left(k^{2}+\Omega^{2}-i \dot{\Omega}\right) X_{k}=0
$$

where $\Omega=\sqrt{q} f$. The background oscillations enter in the form $f(t)=\frac{\phi(t)}{\phi_{0}}$, where $\phi_{0}$ is their amplitude. Without imaginary part, this equation will the the 


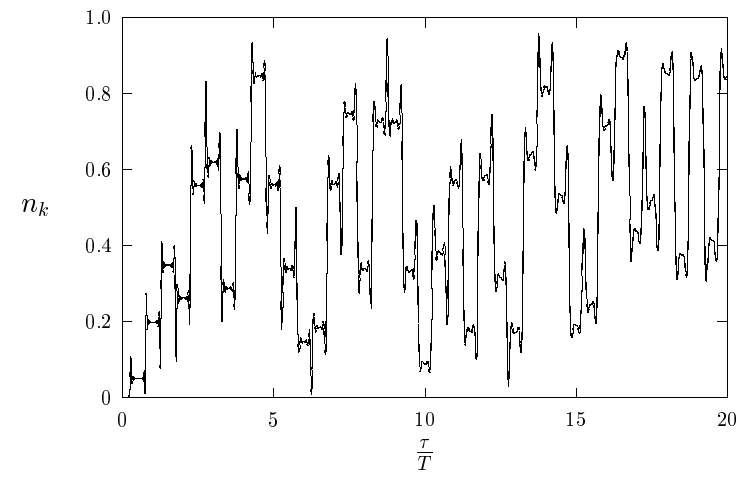

Fig. 7. The occupation number $n_{k}$ of fermions in $m_{\phi}^{2} \phi^{2}$-inflation. The initial resonance parameter is $q_{0}=10^{6}$ and the mode is $\kappa_{0}^{2} \approx \sqrt{q} m^{2}, T=\frac{2 \pi}{m}$.

equation for the fluctuations of the bose field, which results in its parametric resonance. The imaginary part of the frequency in Eq. (15) guarantees the Pauli blocking for the occupation number $n_{k}$. The results for $n_{k}$ can be formulated as follows [2]. Even though the Yukawa interaction contains a small factor $h$, one cannot use the perturbation expansion in $h$. The strength of the effect is ultimately determined by the dimensionless parameter $q$. For instance, for chaotic inflation with $\lambda \phi^{4}$ potential, $q=\frac{h^{2}}{\lambda}$ is typically very large. For instance, in the simple supergravity theory with $W=\sqrt{\lambda} \phi^{3}$ and single chiral multiplet, $h=\sqrt{2 \lambda}$ and $q=2$. For the quadratic inflaton potential $m^{2} \phi^{2}$, the parameter $q=\frac{h \phi_{0}}{M_{p}}$, where $\phi_{0}$ is the amplitude of the inflaton oscillations, which initially is $\sim 0.1 M_{P}^{p}$. The modes get fully excited with occupation numbers $n_{k} \simeq 1$ within tens of oscillations of the field $\phi$, and the width of the parametric excitation of fermions

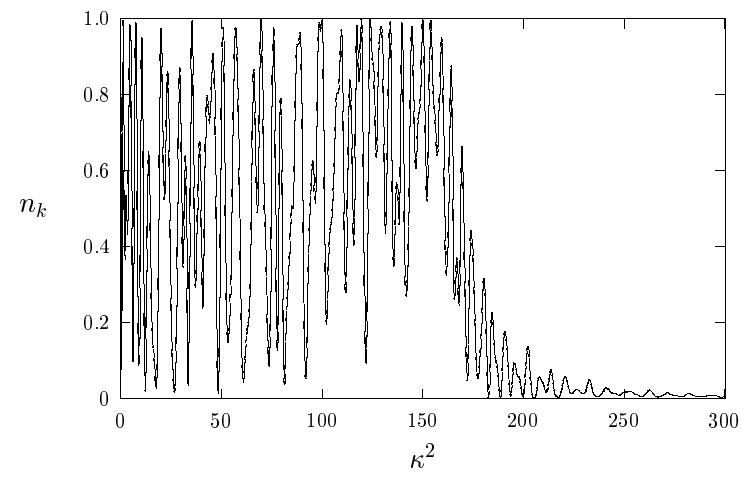

Fig. 8. The comoving occupation number of fermions in $m_{\phi}^{2} \phi^{2}$-inflation after 50 inflaton oscillations for initial resonance parameter $q_{o}=10^{3}$. Expansion of the universe destroys the details of the resonance band and leads to a fermi-sphere of width $q^{1 / 4} \mathrm{~m}$. 
in momentum space is about $q^{1 / 4} m_{e f f}$, where $m_{e f f}$ is the frequency of the background inflaton oscillations. It turns out that the analytic theory of fermionic preheating can be advanced similar to that for the bosonic preheating, see [2] for details. For illustration in Figure 7 we show the time evolution of the occupation number of fermions in $m^{2} \phi^{2}$ inflation, where excitations of fermions is a stochastic process. Figure 8 shows the spectrum of fermionic excitations, which stochastically filling a (Fermi) sphere in the momentum space [2].

\section{Gravitino from Preheating}

Let us now consider the third line in the supergravity Lagrangian (1), which describes the gravitino field $\psi_{\mu}$. Assume that the gravitino mass $m_{3 / 2} \sim 10^{2}-$ $10^{3} \mathrm{Gev}$. Such particles decay very late after nucleosynthesis and lead to disastrous cosmological consequences unless the abundance of gravitinos (in units of the number density of photons) is extremely suppressed, $n_{3 / 2} / n_{\gamma}<10^{-15}$. This puts constrains on the reheat temperature $T_{R}$ to avoid gravitino overproduction from thermal scattering of particles in the very early universe, $T_{R}<10^{8} \mathrm{Gev}$. However, gravitinos can be produced not only in the thermal bath after preheating but even earlier during the inflaton oscillations, similarly to the production of fermions from preheating [3:4]. Thus the investigation of the non-thermal gravitino production in the early universe may serve as a useful tool to discriminate among various versions of inflations.

To study gravitino production from preheating, first one shall derive its equation, which is a challenge by itself. The formalism for gravitinos in an expanding universe and in the presence of complex scalar fields with non-vanishing VEV's was recently formulated in paper [5]. The equation for the gravitino has on the left hand side the kinetic part $R^{\mu} \equiv \epsilon^{\mu \nu \rho \sigma} \gamma_{5} \gamma_{\nu} \mathcal{D}_{\rho} \psi_{\sigma}$, and a rather lengthy right hand side (we use the long derivative $\mathcal{D}_{\mu}$ with the spin connection and Christoffel symbols, for which $\left.\mathcal{D}_{\mu} \gamma_{\nu}=0\right)$. Apart of varying gravitino mass $m_{3 / 2}=\frac{|W|}{M_{p}^{2}}$, the right hand side contains a chiral connection and various mixing terms like those in the 3rd line of (11). For a self-consistent setting of the problem, the gravitino equation should be supplemented by the equations for the fermions $\chi_{i}$ and gauginos $\lambda_{\alpha}$ mixing with gravitino, as well as by the equations determining the gravitational background and the evolution of the scalar fields. We formulated cosmological extension of the super-Higgs effect [5]. It turns out that supersymmetry is spontaneously broken if the combination

$$
H^{2}+m_{3 / 2}^{2}>0
$$

is positive. In flat space-time usually supersymmetry breaking is associated with the non-vanishing gravitino mass $m_{3 / 2}$. In an expanding universe the Hubble parameter $H$ plays an equally important role. For instance, the gravitino mass (i.e. superpotential $W$ ) can vanish, but supersymmetry will be still spontaneously broken. For further discussion of the nonthermal gravitino production, let us make some simplifications. We consider the supergravity multiplet and a single 
chiral multiplet containing a complex scalar field $\Phi$ with a single chiral fermion $\chi$. This is a simple non-trivial extension which allows us to study the gravitino with a non-trivial FRW cosmological metric supported by the scalar field. A nice feature of this model is that the chiral fermion $\chi$ can be gauged to zero so that the mixing between $\psi_{\mu}$ and $\chi$ in (1) is absent. We also can choose the non-vanishing VEV of the scalar field (inflaton) in the real direction. First we will derive the equation for a spin $3 / 2$ field in a curved background metric with non-vanishing VEVs for the scalar fields. From (1) we can obtain the equation for the gravitino We use a plane-wave ansatz $\psi_{\mu} \sim e^{i \mathbf{k} \cdot \mathbf{x}}$ for the space-dependent part. Component $\psi_{0}$ is related to $\psi_{i}$ via the constraint equation. Next, $\psi_{i}$ can be decomposed into its transverse part $\psi_{i}^{T}$, and to the longitudinal part $\psi_{i}^{L}$ which is defined by the trace $\gamma^{i} \psi_{i}$. Two degrees of freedom of $\psi_{\mu}$ are associated with the transverse part $\psi_{i}^{T}$, which correspond to helicity $\pm 3 / 2$ and two degrees of freedom are associated with $\gamma^{i} \psi_{i}$ (or $\psi_{0}$ ) which correspond to helicity $\pm 1 / 2$. Equation for the helicity $\pm 3 / 2$ states for $\mu=i$ is

$$
\left(\gamma^{\mu} \partial_{\mu}+\frac{a^{\prime}}{2 a} \gamma^{0}+m_{3 / 2} a\right) \psi_{i}^{T}=0
$$

The transformation $\psi_{i}^{T}=a^{-1 / 2} \Psi_{i}^{T}$ reduces the equation for the transverse part to the free Dirac equation with a time-varying mass term $m_{3 / 2} a$, c.f. eq. (14). In the previous section we explained how to treat this type of equation. The essential part of $\Psi_{i}^{T}$ is given by the time-dependent part of the eigenmode of the transversal component $X_{T}(t)$, which obeys second-order equation (c.f. (15)):

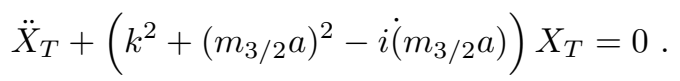

Production of helicity $3 / 2$ gravitino is essential at the fast rolling stage of inflaton evolution, when $W$ may be changed nonadiabatically.

The corresponding equation for gravitino with helicity $1 / 2$ is more complicated. It is convenient to use combination $\theta=\gamma^{i} \psi_{i}$ which fully describes helicity $1 / 2$ states. Equation for combination The equation for $\theta$ is

$$
\left(\partial_{t}+\hat{B}-i \mathbf{k} \cdot \gamma \gamma_{0} \hat{A}\right) \theta=0
$$

where $\hat{B}=-\frac{3 \dot{a}}{2 a} \hat{A}-\frac{m a}{2} \gamma_{0}(1+3 \hat{A})$, and

$$
\hat{A}=\frac{p-3 m^{2} M_{P}^{2}}{\rho+3 m^{2} M_{P}^{2}}+\gamma_{0} \frac{2 \dot{m} M_{P}^{2}}{\rho+3 m^{2} M_{P}^{2}}=A_{1}+\gamma_{0} A_{2} .
$$

Here $\rho$ and $p$ are the energy-density and pressure of the background scalar field. For the single scalar field $|A|^{2}=1$ for an arbitrary superpotential $W$. Thus $A$ can be represented as $A=-\exp \left(2 i \int_{-\infty}^{t} d t \mu(\eta)\right)$, where $\mu=\mathcal{D D} W+\Delta$, the correction $\Delta=\mathcal{O}\left(M_{P}^{-1}\right)$ is given in [5]. The time-dependent factor of the spinor $\theta$, which we denote as $f_{k}(t)$, obeys a second-order differential equation. By 
the substitution $f_{k}(t)=E(t) X(t)$, with $E=\left(-A^{*}\right)^{1 / 2} e^{-\int d t R e B}$, the equation for the function $f_{k}(t)$ is reduced to the oscillator-like equation for the timedependent mode function $X_{L}$ of the longitudinal component:

$$
\ddot{X}_{L}+\left(k^{2}+\Omega_{L}^{2}-i \dot{\Omega}_{L}\right) X_{L}=0
$$

with $a^{-1} \Omega_{L}=\mu-\frac{3}{2} H \sin 2 \int \mu d t-\frac{1}{2} m\left(1+3 \cos 2 \int \mu d t\right)$. Equation 21) describes the creation of gravitinos from preheating. Notable the gravitino with helicity $1 / 2$ remains coupled to the changing background even in the limit $M_{P} \rightarrow \infty$. In a sense, the gravitino with helicity $1 / 2$ remembers its goldstino nature. Thus gravitino production in this background in general is not suppressed by the gravitational coupling. The main dynamical quantity which is responsible for the gravitino production in this scenario will not be the small changing gravitino mass $m_{3 / 2}$, but the mass of the chiral multiplet $\mu$, which is much larger than $m_{3 / 2}$.

But do we really produce gravitinos from preheating, or we describe the production of the chiral fermion? To answer this question, we have to investigate more realistic and more complicated problem with several chiral multiplets. As the first step in this direction we derived equations for gravitinos for an arbitrary number of chiral multiplets plus SYM sector [5]. For instance, generalization of the equation 190 for the next simplest case of two chiral multiplets even in the limit $M_{P} \rightarrow \infty$ is reduced to the complicated second-order spinor equation

$$
\left(\partial_{t}+\hat{B}-i \mathbf{k} \cdot \gamma \gamma_{0} \hat{A}\right) \theta+\hat{G}\left[\partial_{t}^{2}+\left(k^{2}-i \mathbf{k} \cdot \gamma \gamma_{0} \dot{\hat{A}}\right)\right] \theta=0,
$$

the matrix $\hat{G}$ is constructed from the backgroud scalars. We expect solutions of this equations will allow us to find the residual gravitino production. Recent report [9] indicates that helicity $1 / 2$ gravitinos are produced in the model with two chiral multiplets.

\section{References}

1. L. Kofman, A. Linde and A. Starobinsky, Phys. Rev. Lett. 73, 3195 (1994)

2. P. Greene and L. Kofman, Phys. Rev. D62, 123516 (2000)

3. R. Kallosh, L. Kofman, A. Linde, and A. Van Proeyen, Phys. Rev. D61, 103503 (2000)

4. G. Guidice, I. Tkachev and A. Riotto, JHEP 8, 9 (2000)

5. R. Kallosh, L. Kofman, A. Linde, and A. Van Proeyen, Class. Quant. Gravity, 17, 4269 (2000).

6. G. Felder and L. Kofman, hep-th/0011160

7. G. Felder, J. Garcia-Bellido, P. Greene, L. Kofman, A. Linde, I. Tkachev, hep-ph/0012142

8. L. Kofman, in: General Relativity and Relativistic Astrophysics, 8th Canadian Conference (AIP Proceedings 493, 1999), Eds. C. Burgess and R. Myers.

9. D. Lyth and H.B. Kim, hep-ph/0011262 